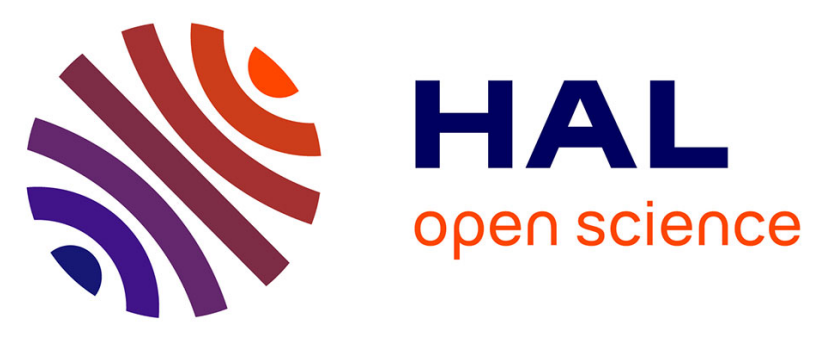

\title{
La Chambre des députés comme ressource et comme théâtre: la consécration de la cause proportionnaliste par le travail en Commission du Suffrage Universel $(1902-1919)$
}

Thomas Marty

\section{To cite this version:}

Thomas Marty. La Chambre des députés comme ressource et comme théâtre: la consécration de la cause proportionnaliste par le travail en Commission du Suffrage Universel (1902 - 1919). Cohen (Antonin), Lacroix (Bernard) et Riutort (Philippe). Les formes de l'activité politique: Eléments d'analyse sociologique (18è - 20è s), Presses Universitaires de France, pp.83 - 101, 2006. halshs00155765

\section{HAL Id: halshs-00155765 \\ https://shs.hal.science/halshs-00155765}

Submitted on 19 Jun 2007

HAL is a multi-disciplinary open access archive for the deposit and dissemination of scientific research documents, whether they are published or not. The documents may come from teaching and research institutions in France or abroad, or from public or private research centers.
L'archive ouverte pluridisciplinaire HAL, est destinée au dépôt et à la diffusion de documents scientifiques de niveau recherche, publiés ou non, émanant des établissements d'enseignement et de recherche français ou étrangers, des laboratoires publics ou privés.

\section{(1) (1) $\$$}

Distributed under a Creative Commons Attribution - NonCommercial - NoDerivatives 44.0 


\section{Thomas Marty (GAP - Paris X)}

«La Chambre des députés comme ressource et comme théâtre : la consécration de la cause proportionnaliste par le travail en Commission du Suffrage Universel (1902 1919) » in Antonin Cohen, Bernard Lacroix et Philippe Riutort (dirs), Les formes de l'activité politique : Eléments d'analyse sociologique, Paris, PUF, 2006, p 83 - 101.

Résumé : La croyance en l'existence d'un «bon » mode de scrutin n'a pas toujours existé. Cette étude revient sur les avatars et les errements qui séparent, dans les années 1900, le moment à partir duquel des parlementaires se font les avocats d'un système alternatif au scrutin majoritaire uninominal d'arrondissement alors en place, et connu sous le nom de «R.P» (représentation proportionnelle), et le moment où la majorité parlementaire se rallie à ce nouveau mode de scrutin en 1919. L'investigation historiographique relative au changement de la règle électorale présente très souvent celle-ci, soit comme l'expression d'une «intention » modernisatrice soit comme le produit de stratégies partisanes intéressées. Mais la transformation du savoir électoral en objet et en enjeu politique induite par la revendication d'une nouvelle forme d'équité fonctionne plutôt comme une stratégie pragmatique d'affirmation d'une Commission parlementaire du Suffrage universel. La consolidation de la collaboration entre des parlementaires et des spécialistes du travail symbolique en politique qu'on appelle alors de façon indigène des «publicistes » (journalistes, juristes, mathématiciens) est à l'origine de la vulgarisation de la cause proportionnaliste et met en valeur la fonction centrale de cette commission.

Mots clefs : mode de scrutin, réforme électorale, parlement, publicistes, action collective.

Existe-t-il un bon mode de scrutin ? Ou du moins, existe-t-il une quelconque hiérarchie entre les diverses modalités contemporaines du «faire voter»? Olivier Dassault, actuel député UMP de l'Oise semble le croire en des termes qui a posteriori peuvent apparaître comme assez proches de ceux dont se servent les partisans de la représentation proportionnelle $(\mathrm{RP})$ au début du $20^{\text {ème }}$ siècle : «L'ampleur de la défaite de la droite aux régionales peut-elle permettre de revenir sereinement au débat sur le bon mode de scrutin dans ce pays ? [...] au-delà de ses aspects purement électoraux, le mode de scrutin a un effet structurant sur la vie démocratique et [...] il n'est pas étranger à la «volatilité» dont fait preuve notre électorat $[\ldots] »^{1}$. Pourtant la croyance en l'existence d'un «bon» mode de scrutin comme aussi la croyance en l'existence d'une hiérarchie entre ces modes de scrutin disponibles n'a pas toujours existé. Il a fallu tout d'abord pour qu'elle prenne forme que se spécifient des modes de scrutin concurrents et que leurs particularités comme leurs éventuels effets soient clairement identifiés. Il a fallu également que les formes les moins établies soient connues et reçues au sein du monde parlementaire avant qu'elles ne deviennent enjeu de luttes entre parlementaires. C'est justement sur cette séquence et ce qui s'y joue qu'on voudrait

\footnotetext{
${ }^{1}$ Olivier Dassault, «L'isoloir ne doit pas être un défouloir », Le Figaro, 5 avril 2004.
} 
revenir : sur les avatars et les errements qui séparent, dans les années 1900, le moment à partir duquel des parlementaires se font les avocats d'un système encore mal stabilisé, et connu sous le nom de «R.P », et le moment où la majorité parlementaire se rallie à ce nouveau mode de scrutin en 1919.

Tout ne semble pas en effet avoir été dit du travail collectif nécessaire jusqu'à ce que ce nouveau mode de scrutin soit apprivoisé et consacré. Les historiens nous ont certes restitués les débats majeurs en séance plénière dans les années qui précédent la première guerre ${ }^{2}$. Ils ont également identifiés la propagande, initiée par quelques publicistes, autour de cette idée neuve $^{3}$. Mais ils laissent supposer, conformément à la vision établie de la relation entre le peuple et ses représentants que ce nouveau mode de scrutin a été imposé aux parlementaires pour l'essentiel «de l'extérieur ». Ils négligent ce faisant la conversion propre à de nombreux parlementaires notamment sous l'effet des débats propres à la Commission du Suffrage Universel (CSU). En ratifiant implicitement une version idéalisée du fonctionnement de la démocratie, ils passent ainsi sous silence l'institution au sein du parlement d'une « institution dans l'institution » qui joue un rôle capital dans la publicité de ce nouveau mode de scrutin. En revenant aux propriétés politiques et intellectuelles des membres de cette commission et au travail de mise en circulation de la «R.P » qu'elle organise, qu'elle centralise et qu'elle coordonne, en se servant de toutes les ressources du jeu parlementaire (interventions orales, propositions de loi, rapports et amendements), on ne fera pas seulement réapparaître une débauche d'activités oubliées, on fera voir aussi que la neutralisation et la naturalisation de cette idée sont autant de conditions pratiques indispensables à sa consécration juridique sous le sceau de l'autorité parlementaire.

L'investigation historiographique relative au changement de la règle électorale, focalisée sur l'issue parlementaire présente très souvent celle-ci, soit comme l'expression d'une «intention» modernisatrice ${ }^{4}$ soit comme le produit de stratégies partisanes intéressées ${ }^{5}$ : elle fait donc voir dans tous les cas ce changement de façon finaliste en concevant implicitement l'établissement du système partisan qui se révèle après coup, comme le télos de la

\footnotetext{
${ }^{2}$ Raymond Huard, Le suffrage universel en France 1848 - 1946, Paris, Aubier, 1991, p 161 - 185.

${ }^{3}$ Gilles Le Béguec, «La représentation proportionnelle. Cent ans de controverses », Vingtième Siècle, 1986, n 9, p $67-80$.

${ }^{4}$ Serge Noiret, «Les réformes électorales de 1918 - 1919 en Italie : révolution démocratique ou cause de l'avènement du fascisme », Annie Laurent, Pascale Delfosse et André Paul Frognier, Les systèmes électoraux : permanences et innovations, Paris, L'Harmattan, 2004, p 185 - 215.

${ }_{5}$ Pascale Delfosse, «Les changements de mode de scrutin en Belgique», dans A. Laurent et al. (dir.), Les systèmes électoraux, Op. Cit, p $151-184$.
} 
transformation de la règle électorale. L'enquête dans les archives ${ }^{6}$ met seulement en présence, très prosaïquement, d'une conjoncture particulière de professionnalisation de la vie politique qui s'accompagne d'une division du travail accrue, coïncidant avec l'apparition d'une offre d'expertise électorale. En cela la réforme électorale apparaît moins comme la manifestation d'une crise supposée de la représentation propre à la $3^{\text {ème }}$ République d'avant guerre (et qui serait récurrente depuis) ${ }^{7}$ que comme un moment d'élargissement, de consolidation et d'autonomisation accrue d'un milieu partisan en voie de structuration ${ }^{8}$. La transformation du savoir électoral en objet et en enjeu politique (inhérente à la quantification et la classification des modes de scrutin, des partis et des candidats) induite par la revendication d'une nouvelle forme d'équité fonctionne ainsi comme une stratégie pragmatique d'affirmation de la CSU. Centre d'impulsion d'un action collective, point de repère de l'action d'auxiliaires intéressés à la vie parlementaire et partisane (journalistes, publicistes, élus locaux, professeurs de mathématiques) poursuivant chacun pour leur compte des objectifs différents, la CSU devient le point de coordination pratique d'organes et d'intérêts expressifs séparés et souvent antagonistes, apparus le plus souvent antérieurement et extérieurement à son action effective. Bien loin que la représentation soit un résultat attaché à l'existence de «l'institution parlementaire », elle n'est qu'une ressource symbolique supplémentaire d'affirmation de la prétention des parlementaires au monopole de la politique.

Ce point de vue et cette perspective conduisent ainsi à décrire la position et la place particulière de la CSU dans l'univers des relations entre groupes interdépendants conscients des ressources que donne une position parlementaire mais ignorants des distances et des frontières qui paraissent, aux yeux de l'observateur a posteriori les séparer. On suivra ensuite les manifestations et les effets les plus explicites de l'usage parlementaire d'un savoir expert en matière électorale. La codification finale des règles de la compétition entre partis peut être considérée au total comme une expression des transformations du recrutement parlementaire et du crédit accru des images publiques de la délégation électorale.

\section{L'institution parlementaire de la cause « proportionnaliste »}

\footnotetext{
${ }^{6}$ Ce travail repose sur l'exploitation des archives de la commission du suffrage universel entre 1902 et 1919 conservées aux Archives Nationales sous les cotes C 7375, C 7447 et C7721.

${ }^{7}$ Sur la confusion du réel et de sa mesure, voir Bernard Lacroix, «La « crise de la démocratie représentative » en France. Eléments pour une discussion sociologique du problème », Scalpel, 1994, nº 1, p 6 - 29.

${ }^{8}$ Ce moment se prolongeant dans les années 20 sur d'autres terrains de réforme électorale. Voir Guillaume Marrel et Renaud Payre, «Le temps des maires. L'allongement du mandat en 1929 : une redéfinition de l'espace politique municipal », Politix, 2001, n॰53, p $59-86$.
} 
Le procès de légitimation par la Commission du Suffrage Universel

Le travail en commission comme condition de l'accès différentiel des hommes et des idées à la publicité devient le point de coordination des mobilisations étrangères les unes aux autres qui fondent la cause proportionnaliste. La vulgarisation de «savoirs électoraux » qui s'opère à partir de la CSU dépend ainsi directement de la position qu'elle occupe et du rôle qu'elle joue dans la réorganisation du travail de propagande en faveur de la proportionnelle. En tant que travail collectif, le travail parlementaire donne à voir une dynamique dotée de son tempo spécifique mais aussi des effets de cliquet indispensables à la réussite de thématiques qui n'ont rien à voir avec les enjeux de la construction de l'initiative qui en devient le support. Bien que le projet remonte au début de la précédente législature ${ }^{9}$, la CSU se met en place en 1902 et ne joue qu'un rôle restreint au moment où la mobilisation des parlementaires prend forme. Il n'est ainsi paradoxal qu'en apparence, à raison même des effets pratiques de proximité et de réseau au sein de l'espace des prétendants aux fonctions gouvernementales que la commission devienne assez vite «l'interlocuteur» légitime des gouvernements successifs lorsque la RP devient une cause «publique ». C'est ainsi pendant le ministère radical Clemenceau que la commission tend à se confondre avec la cause proportionnelle en un Groupe Parlementaire pour la Réforme Electorale, organisant au même moment à travers toute la France des dizaines de réunions publiques en direction de «l'opinion ». Présidées par le député de la Fédération Républicaine de Paris, Charles Benoist ${ }^{10}$, ces deux figures publiques apparemment distinctes, apparaissent comme deux incarnations d'un même groupe militant engagé dans la lutte contre le rejet gouvernemental de principe de toute introduction de la RP. Inversement, mais pour les mêmes raisons structurelles, lorsque le cabinet Briand succède au cabinet Clemenceau jusqu'en 1911, la CSU devient le théâtre de l'organisation de la résistance au projet «transactionnel » de « représentation des minorités » présenté par le président du conseil ${ }^{11}$. C'est ainsi lorsque l'initiative change de camp et revient au gouvernement que la commission parlementaire devient le commandement opérationnel visant à sauvegarder la pureté des intentions doctrinales de la réforme. En même temps, l'attention au plus immédiatement apparent que le juriste spécialiste d'institutions serait tenté de lire en termes de dissensions entre «le pouvoir exécutif» et le «pouvoir législatif » ne

\footnotetext{
${ }^{9}$ Alain Garrigou, Histoire sociale du suffrage universel. 1848 - 2000, Paris, Seuil, 2002, p 193.

${ }^{10}$ Charles Benoist, Souvenirs, Tome 3, Paris, Plon, 1934, p 153 et suivantes.

${ }^{11}$ Journal Officiel (JO), Documents parlementaires (DP), 18 Août 1910, n 201.
} 
saurait dissimuler l'espèce de doxa qui s'est formée entre parlementaires sur la question : le problème abstrait et mathématisé de la représentation qui résultait des controverses antérieures et qui les caractérisait est repris et reformulé conformément aux enjeux propres du métier parlementaire. Il s'efface ainsi au profit de la recherche de ressources étatiques seules à même de produire l'expertise de nature à résoudre les contradictions dûment explicitées de la représentation. Quelle est la «bonne taille des circonscriptions » ? Que saiton exactement de la relation entre inscrits et votants ? Comment peut-on classer des candidats de façon incontestable ? Est-il possible de se faire une idée anticipée des scores ? A. Briand se fait l'expression de cette transformation des façons de voir lorsqu'il se présente devant la commission pour affirmer que «... tout en poursuivant cet idéal de justice mathématique [les partisans de la RP], ont dû céder en même temps à des préoccupations de géographie politique $»^{12}$.

Division du travail de propagande en faveur de la proportionnelle

La transformation (et la consolidation) de la collaboration entre des parlementaires et ces spécialistes du travail symbolique en politique qu'on appelle alors de façon indigène des « publicistes » (journalistes, juristes, mathématiciens) qui est à l'origine de la vulgarisation de la cause proportionnaliste met en valeur la fonction centrale de la commission. Il n'existe ainsi encore en 1902 qu'une seule organisation commune aux députés et aux promoteurs de la cause qui ne sont pas professionnellement intéressés à celle-ci : la Ligue pour la Représentation Proportionnelle. Les députés intéressés par la question sont plutôt animés de préoccupations générales à l'instar de C. Benoist, E. Flandin, L. Mill. Ils sont à la fois animateurs de la CSU et membres de la Ligue où ils rencontrent les spécialistes de la cause «intellectuelle» de la RP (PG. La Chesnais, E. Macquart, J. Dessaint). Les travaux alors produits sont reconnus comme des références cardinales dans la controverse militante même s'il s'agit de reprises de travaux législatifs antérieurs. Le rapport de C. Benoist en $1905^{13}$ est proposé en volume à grand tirage, et il doit à cette propriété de devenir jusqu'en 1911 l'un des principaux bréviaires historique et juridique des proportionnalistes ${ }^{14}$. Dans le même sens, le rapport et la proposition du député de l'Yonne Etienne Flandin ${ }^{15}$ serviront de lien aux réunions du Groupe Parlementaire de 1908 à 1909 : il a commencé son existence publique

\footnotetext{
${ }^{12}$ Archives Nationales (AN), C 7447, 22 Décembre 1910.

${ }^{13}$ JO, DP, 20 Juillet $1905, \mathrm{n}^{\circ} 2376$.

${ }_{14}^{14}$ C. Benoist, Pour la réforme électorale, Paris, Plon, 1908.

${ }^{15}$ JO, DP, n 883, 22 Mars 1907 ; n 408, 27 Avril 1907.
} 
sous l'espèce de tracts de synthèse avant de devenir un opuscule publié ${ }^{16}$ après avoir servi de référence à l'éloquence de la tribune militante. Le caractère unifié de cette coopération se manifeste même par une proposition de loi conçue par la Ligue et déposée par le député de la seine L. Mill ${ }^{17}$.

A partir de 1910, cette mobilisation prend des formes sensiblement différentes. Il n'est plus nécessaire de mobiliser de capital au sein du parlement puisque son crédit fait de la question des techniques de désignation des députés une cause publique légitime. Les députés peuvent donc se contenter d'assurer une présence et une publicité en dehors de l'enceinte parlementaire quoique cette forme d'action constitue désormais aussi une façon pour des prétendants d'afficher leurs ambitions ultérieures. Le comité extra-parlementaire de la RP n'a pas d'autre raison d'être : il est présidé par l'avocat Henry Lémery ; il est animé par d'anciens parlementaires ; il est enfin tiré par plusieurs aspirants parlementaires encore au stade militant de leur investissement. Surtout, le capital proprement intellectuel indispensable dans un premier temps à la consécration parlementaire de la revendication proportionnaliste n'est plus aussi nécessaire que dans la configuration antérieure. Désormais, les soutiens «intellectuels » (universitaires et académiciens) sont renvoyés à des formes de mobilisation plus «autonomes »: le comité républicain de la RP sous la présidence d'Adolphe Carnot, autorité intellectuelle indiscutable en la matière, mais animé en fait par le journaliste et publiciste Georges Lachapelle. Le porte-parole public de ce comité est une commission d'études - le nom n'a rien d'indifférent- qui rassemble la plupart des membres de l'ancienne Ligue pour la RP. L'orientation sensiblement différente, plus nettement pratique et politique d'un côté (sur des positions de défense des formes constituées de la politique) et plus proprement intellectuelle de l'autre (sur des positions plus proches des enjeux citoyens de la «République »), et en un sens plus « universel»s'exprime dans les modes d'action et de mobilisation adoptés. Le comité républicain persévère dans des formes de mobilisation confiantes dans le pouvoir de convaincre comme l'atteste le livre que G. Lachapelle fait paraître en $1911^{18}$ introduit par le mathématicien Henri Poincaré et qui remplacera le Pour la réforme électorale de $\mathrm{C}$. Benoist, ou bien la brochure La représentation proportionnelle des partis politiques $^{19} \mathrm{du}$ même Lachapelle qui renoue avec la tactique de propagande dont la

\footnotetext{
${ }^{16}$ Etienne Flandin, La représentation proportionnelle. Mécanisme et fonctionnement, Paris, Le soudier, 1910.

${ }^{17} \mathrm{JO}, \mathrm{DP}, \mathrm{n}^{\circ}$ 967, 29 Juin 1903.

${ }^{18}$ Georges Lachapelle, La représentation proportionnelle en France et en Belgique, Paris, Félix Alcan, 1911.

19 G. Lachapelle, La représentation proportionnelle des partis politiques, Paris, Publications du comité républicain de la $\mathrm{RP}, 1913$.
} 
Ligue avait tirée partie mais cette fois sous la caution politique et scientifique d'un préfacier dépositaire de la double autorité scientifique et politique: Adolphe Carnot (ingénieur des mines, membre de l'Académie des sciences et président de l'Alliance Démocratique) ${ }^{20}$. A l'opposé, le comité extra-parlementaire s'associe à la campagne de conférences que le Groupe Parlementaire reprend en Décembre 1909. Il s'appuie sur l'autorité politique de conférenciers qui aspirent pour beaucoup d'entre eux à devenir députés ou qui seront bientôt contraints de quitter la scène parlementaire après une défaite électorale (comme le rapporteur de la CSU, A. Varenne, en 1910). A ce titre, la cause proportionnaliste est vers 1910 un élément de l'avantgardisme en politique : mais la dissociation de ses partisans que rend possible l'accord acquis sur cet objet de désaccord politique laisse voir toute la distance qui sépare l'utopie politique d'intellectuels désintéressés de la politique à cause de leurs investissements scientifiques comme l'analyse mathématique ou à partir de revendications réformatrices comme le système d'Hondt inspiré de la réforme électorale belge et la perspective politique de professionnels de la politique intéressés à la promotion d'un avenir qui passe par l'élection, la valorisation du capital rhétorique de l'éloquence inspirée et l'avancement dans le cursus honorum du représentant tel que celui-ci se redessine alors.

\section{Codes, coups et croyances dans la controverse proportionnaliste}

On aurait ainsi tort de réduire la question électorale et la question du mode de scrutin qui l'exprime à un problème intellectuel abstrait, à un problème strictement technique ou même à un simple problème d'efficacité ou d'inefficacité parlementaire étroitement fonctionnel. Il y va aussi à travers la circulation et la diffusion de bribes de savoir électoral ${ }^{21}$ de l'universalisation de l'image que les hommes politiques se font de leur rôle et corrélativement du contrôle qu'il leur paraît légitime d'exercer sur leur nomination : pour que soit disqualifié à défaut d'être invalidé toute forme de dénégation de leur droit à parler au nom de tous dans un monde social et politique qui se transforme. Trois déplacements majeurs font ainsi émerger les nouveaux «codes » et les nouvelles «disciplines » de l'analyse anticipée des faits et des

\footnotetext{
${ }^{20}$ Rosemonde Sanson, «Adolphe Carnot et l'Alliance républicaine démocratique », dans G. Le Béguec (dir.), Une lignée républicaine : les Carnot sous la IIIè République, Limoges, Lucien Souny, 1989, p 85 - 102.

${ }^{21}$ La notion de «savoir électoral » entend désigner l'ensemble des outils intellectuels de l'analyse anticipée des élections. Elle participe d'une division du travail électoral qui, comme l'a montré E. Phélippeau à propos des savoir-faire de l'élection, engendre la structuration progressive de nouveaux acteurs individuels et collectifs. Voir Eric Phélippeau, L'invention de l'homme politique moderne. Mackau, l'Orne et la République, Paris, Belin, 2002, chapitres 3 et 6 .
} 
effets électoraux. Tout part de «l'anarchie» qui semble caractériser la perception des classements partisans induit par la dynamique endogène du fonctionnement électoral. Tout paraît, en conséquence, passer par la maîtrise pratique d'un sens statistique des «bonnes proportions » en matière d'activité politique. La recomposition du mode de classement des scrutins et la pertinence de la qualification juridique de leurs effets politiques sont le résultat final de ces évolutions.

Inflation et diversification des classements partisans

On ne peut pas ignorer en premier lieu la situation définie par l'inflation des classements partisans produits à l'occasion de la présentation et de l'interprétation des résultats électoraux. Tout se passe comme si les membres de la CSU étaient écartelés entre deux formes de classement qui ont leurs titres, leurs mérites et leurs partisans : ceux des membres de l'administration préfectorale, appuyés sur leurs connaissances locales et ceux des auxiliaires des partis prompts à rechercher au-delà de l'émiettement géographique le sens national de l'opération électorale ${ }^{22}$. Les députés de la CSU se retrouvent donc dans une situation où, proposant leurs classements à des fins d'action législative, ils sont contraints de se situer «au dessus » et «au-delà » des taxinomies disponibles, où si l'on préfère, de «bricoler » une rationalité métapolitique à géométrie variable qui indique seulement qu'ils sont moins portés à se classer sur un continuum partisan qu'à prendre parti à partir d'une échelle partisane qui donne elle-même une consistance au classement distinctif des partis. La distance sociale et professionnelle des députés par rapport aux membres du corps préfectoral couplée à un recours croissant aux ressources administratives pour «penser» la réforme électorale expliquent dans un premier temps les différentes variations autour de la «valence positive ou négative ${ }^{23}$ des partis ou des «blocs» qui permettent de justifier ou de condamner l'introduction de la RP. L'universalisation mathématique de catégories administratives duales agit comme une technique sui generis dans la controverse proportionnaliste. L'exemple du député Alexandre Vazeille, membre «historique » de la Ligue pour la RP dés 1901, permet d'expliquer son éloignement de la RP à partir d'un usage intensif de cette «dualité »: «Il craint un projet qui n'est proportionnel que de nom et risque de créer des interversions de majorité et de minorité [...]. Ainsi si l'on prend 90000 inscrits et 80000 votants : 4 sièges et

\footnotetext{
${ }^{22}$ Michel Offerlé, «Le nombre de voix. Electeurs, partis et électorat socialistes à la fin du $19^{\text {ème }}$ siècle en France », Actes de la Recherche en Sciences Sociales, 1988, n $71-72$, p 5 - 21.

${ }^{23}$ Eric Phélippeau, «La fabrication administrative des opinions politiques. Votes, déclarations de candidature et verdict des préfets (1852 - 1914) », Revue Française de Science Politique, 1993, n4, p 587 - 612 (p 600).
} 
3 listes. Voix républicaines en 2 listes: A $(33$ 000) et $\mathrm{B}(11000)=44000$. Voix d'opposition : C (36 000). Avec le système d'Hondt, les voix républicaines n'ont que deux sièges contre deux sièges à l'opposition. [...] S'il est équitable de donner leur part aux conservateurs, il est inadmissible de leur donner plus que leur part $»^{24}$. Toutefois, c'est en partant des propriétés des agents partisans affectés à cette fonction de classement que l'on peut saisir la transformation fondamentale opérée par cet alignement des députés sur les travaux de publicistes auxquels ils ont initialement donné du crédit. Par exemple, PG. La Chesnais et G. Lachapelle présentent la double particularité d'avoir accumulé un fort capital militant et intellectuel en matière de RP et d'être inclus dans des appareils partisans plus ou moins structurés. Les usages disparates de ces deux «auteur - acteur» par les différents commissaires permettent de sortir du cercle selon lequel les intervenants se retranchent derrière les faits : les statisticiens lorsqu'ils enregistrent les opérations de regroupement des entreprises de mobilisation électorale; les députés lorsqu'ils paraissent endosser ces travaux sans les amender. La genèse de cette statistique électorale se comprend d'abord en relation avec les conjonctures électorales qui l'ont vu naître. Aux élections de 1902 et 1906, seul PG. La Chesnais (préposé à la prospective proportionnaliste au sein du parti socialiste) participe à cette production essentiellement de manière incidente pour appliquer les modes de calcul du système d'Hondt à la réalité sortie des urnes. Il est ainsi rétrospectivement bien difficile de distinguer son travail de la logique partisane qui a présidé, entre ces deux élections, à la constitution du bloc des gauches : il passe en effet d'un classement Socialistes / Radicaux / Républicains / Réactionnaires ${ }^{25}$ à une échelle topographique ${ }^{26}$ Socialistes / Socialistes indépendants / Radicaux / Radicaux indépendants / Républicains de Gauche / Progressistes / Conservateurs $^{27}$. La projection de l'unité de l'appareil partisan socialiste sur les familles radicales explique la précocité de la diffusion du réflexe «blocard ${ }^{28}$ et plus tardivement l'alignement des députés de ces formations sur les schémas produits par le seul PG. La Chesnais. La codification d'une échelle de classement partisan prend donc d'abord forme au titre de maîtrise par les députés de l'image et de la stylisation statistique de leur propre coalition. L'entrée en scène, à partir de 1910, de G. Lachapelle au côté de PG. La Chesnais modifie cette maîtrise inégalement répartie mais du point de vue de son audience. Homme

\footnotetext{
${ }^{24}$ AN, C7447, 7 Décembre 1910.

${ }^{25}$ Pierre Georget La Chesnais, La représentation proportionnelle et les partis politiques, Paris, Société nouvelle de librairie et d'édition, 1902, p 53.

${ }^{26}$ Nous reprenons le terme introduit par E. Phélippeau, « La fabrication administrative ... », Art. Cit, p 600.

${ }^{27}$ PG. La Chesnais, « Statistique électorale », Revue de Paris, 15 Juin 1906, p 866 - 894 (p 879).

${ }^{28}$ Connoté péjorativement, le terme n'en a pas moins servi à formaliser l'unité du bloc des gauches.
} 
d'appareil lui aussi aux alentours de 1900, mais au centre-gauche ${ }^{29}$, G. Lachapelle reste un entrepreneur de presse et de cause dont le ralliement tardif à la RP vers 1909 / 1910 est pour beaucoup dans la reconfiguration collective de cette cause. Ce qui chez La Chesnais relevait d'une prospective partisane à peine masquée derrière l'argumentaire proportionnaliste, devient chez Lachapelle, au regard de ses échecs passés (électoral et gouvernemental) ${ }^{30}$, la nécessité de lutter pour un sens pratique plus aigu du classement partisan. En associant le quotidien «Le Temps » et le vade-mecum «L'annuaire du parlement » à cette entreprise de publication et de systématisation des résultats électoraux ${ }^{31}$, l'action de Lachapelle peut être considérée comme le chaînon manquant entre la «politisation de savoirs électoraux » par la CSU et l'écho de la campagne proportionnaliste dans des cercles sociaux étendus.

Les «bonnes proportions » de l'activité politique

Parmi les autres variables de différenciation, celles qui posent la question préjudicielle des «bonnes proportions » de l'activité politique ne sont pas les moins controversées. En posant comme un double problème jamais résolu la question de la base électorale (inscrits, électeurs ou population globale) et celle du périmètre pertinent de l'élection (découpage des circonscriptions et nombre de députés par unité de territoire et/ou de population), les adversaires explicites de la RP prétendent ainsi faire plus «proportionnel» que les «proportionnalistes». Les propositions et rapports de JL Breton ${ }^{32}$ sont, par exemple, traversé(e)s par l'idée d'accorder un nombre de députés par département proportionnel à leur population. La conservation du scrutin uninominal majoritaire associée à un intense travail de «péréquation des circonscriptions électorales » par rapport à la loi de 1889 appuyée sur les arrondissements aboutissent à ce que ce député nommera la représentation proportionnée. L'importance du travail statistique fournit par Breton ${ }^{33}$, quand bien même il ne s'est inscrit

\footnotetext{
${ }^{29}$ Secrétaire général de l'Union Libérale Républicaine fondée par Léon Say. Voir G. Le Béguec, «Le libéralisme et les défis de l'action collective », Mélanges de l'Ecole Française de Rome, Série Italie Méditerranée, 2002, n², p $745-759$.

${ }^{30}$ A la différence de La Chesnais, la trajectoire de Lachapelle est marquée par un échec aux législatives (en 1902). Les conditions de cette lutte électorale où, face à deux candidats radicaux il est contraint de se situer sur un espace politique qu'il ne reconnaît pas, expliquent son investissement dans la production de telles classifications. Voir les dossiers conservés aux Archives Départementales de Dordogne : 3 M 73, 4 M 17.

${ }^{31}$ PG La Chesnais et G. Lachapelle, Tableau des élections législatives des 24 Avril et 8 Mai 1910, Suivi d'une application de la représentation proportionnelle - système rationnel et système d'Hondt, Paris, G. Roustan - Le Temps, 1910.

${ }^{32} \mathrm{JO}, \mathrm{DP}, \mathrm{n}^{\circ} 1989,10$ Juillet 1908.

${ }^{33}$ On trouvera dans les papiers C. Benoist une version entièrement annotée des tableaux de péréquation proposés par Breton prouvant l'intérêt que le chef de file des proportionnalistes accordait à son adversaire membre de
} 
dans aucune perspective d'aboutissement législatif, fait de l'activité d'un des opposants les plus acharnés à l'idée de représentation proportionnelle un élément tout à fait fondamental de la visibilité publique de la controverse elle-même, notamment du côté des rationalisations intéressées de cette controverse. Dans la même optique, l'amendement proposé par le député meusien de la Gauche démocratique André Maginot ${ }^{34}$ d'élire un député pour 25000 électeurs et non plus un député pour 70000 habitants, met en valeur les différenciations des représentations de la délégation et entérine, par exemple, la distinction entre les catégories analytiques de «droit électoral» et de «mode de scrutin» (qui ne produirait ses effets que par et pour les électeurs). Jaurès lui-même, pourtant favorable au droit de vote des femmes, considèrera comme une «représentation indirecte» des femmes le fait que le député soit le délégué des habitants et non simplement des électeurs ${ }^{35}$.

\section{La classification des modes de scrutin}

Les croyances investies en termes de classification des modes de scrutin ne font que résumer et synthétiser sous une forme économique ces transformations. Etienne Flandin, membre de la Ligue et premier rapporteur dont le travail aboutira à un débat en séance, contribua ainsi à synthétiser devant la CSU, dés le début de la législature 1906 - 1910, une échelle de classification appelée à devenir la matrice de toutes les autres. Il relève quatre systèmes de représentation proportionnelle : «belge sans panachage des listes » (système d'Hondt qui consiste à rechercher un diviseur commun entre toutes les listes) ; «belge amendé avec panachage demi-toléré »; «proposition Dansette » (système du quotient qui consiste à décider a priori du nombre de voix nécessaire pour élire un député et d'attribuer les sièges restants aux plus forts restes); et enfin la propre proposition de Flandin (qui reprend le système d'Hondt mais avec un panachage total puisque le vote porte sur un candidat qui aura préalablement choisi sa liste d'affiliation) ${ }^{36}$. Son travail est en fait la figuration de l'éventail des possibles politiques qui balisera intellectuellement les divergences entre les membres de la CSU jusqu'en 1919. Il agit par sa partialité même comme l'origine d'un débat produisant ses «codes » et ses «coups » partagés aussi bien par les partisans que par les adversaires. Si l'échelle de Flandin aménage les conditions de la différenciation entre partisans de la RP

l'Académie des sciences. Voir Bibliothèque de l'Institut de France, Manuscrits Charles Benoist, Mss 4534, dossier 4.

${ }_{34}^{34}$ AN, C 7447, $1^{\text {er }}$ Février 1911.

${ }^{35}$ Ibid.

${ }^{36}$ AN, C 7375, 14 Novembre 1906. 
(savoir si la proportionnalité doit relever d'un quotient pré-défini ou établit de manière ad hoc, savoir si une liste peut être modifiée par l'électeur ou non), elle ménage tout autant le débat sur les autres formes de scrutin au moins en tant que rappel à l'ordre dans les moments d'enlisement du débat. Ainsi, au moment d'émettre un vote de principe sur la RP et face à la neutralité feinte de plusieurs commissaires, le député mathématicien Paul Painlevé affirme : «L'opposition des systèmes se fait ainsi: scrutin d'arrondissement, scrutin de liste majoritaire, scrutin de liste avec RP»; ce qui lui vaut une réponse de $\mathrm{C}$. Benoist : «Il y a deux coupures de systèmes bien tranchées: système majoritaire et variation de proportionnalité dans ses différents modes $»^{37}$.

\section{L'improbable codification des règles de la compétition inter-partisane}

On comprend le rôle central de cette commission parlementaire au regard d'une conjoncture qui la place au centre de réseaux mobilisés et qui lui assure ainsi de pouvoir politiser cette revendication. Il ne reste plus qu'à tenter de préciser la portée pratique d'un travail de codification qui n'avait encore fait ni l'objet d'un investissement parlementaire ni l'objet d'un travail de formalisation de la part de tous ceux qui jouent le rôle d'auxiliaire de l'activité politique. Il est clair en effet que si l'organisation pratique et matérielle du vote avait déjà fait l'objet bien avant la décennie 1909 - 1919 d'un effort collectif d'explicitation ${ }^{38}$, les conditions de la compétition partisane elle-même étaient jusqu'alors restées en dehors de cette sphère d'intervention. Or, elles sont désormais liées l'une à l'autre à travers la présence et la circulation d'un même personnel parlementaire. On connaît par exemple l'investissement du député des Basses Alpes Joseph Reinach dans l'adoption de la loi de Juillet 1913 sur le secret et la liberté du vote en tant que rapporteur mais aussi en tant que vice-président de la CSU. N'intervenant que très épisodiquement dans le débat sur la RP, il manifeste cependant au plus haut point la dissonance entre la latitude d'action que la commission s'octroie et la croyance, sans cesse renouvelée, dans sa capacité à réaliser la réforme électorale : « Ce n'est pas parmi ceux d'entre nous qui depuis quelques années ont soutenu le principe de la proportionnelle avec le plus de passion que vous trouverez le plus d'intransigeance, parce que nous avons

\footnotetext{
${ }^{37}$ AN, C 7447, 19 Octobre 1910.

${ }^{38}$ Sur le suffrage universel comme mobilisation d'Etat, cf. Bernard Lacroix, « Retour sur 1848. Le suffrage universel entre l'illusion du «jamais vu » et l'illusion du «toujours ainsi » », Actes de la Recherche en Sciences Sociales, $\mathrm{n}^{\circ}$ 140, Décembre 2001, p 41 - 50 ; A. Garrigou, Histoire sociale du suffrage universel, Op. Cit, p 141 - 217 ( « les règles du jeu électoral »).
} 
beaucoup étudié cette question et que nous nous sommes rendu compte qu'on n'arrivera jamais à la représentation mathématiquement exacte mais seulement à des approximations $[\ldots] \gg{ }^{39}$.

Il n'est pas sans intérêt de s'attarder sur les propriétés individuelles et collectives qui éclairent la perpétuation de ce qu'on croît être après coup un débat et qui apparaît se réduire à sa difficulté intrinsèque à trouver une issue avant le début de la guerre. Un des parlementaires les plus investis dans cette perpétuation de la controverse est sans aucun doute Arthur Groussier, ingénieur dessinateur industriel et rapporteur socialiste de la Commission du Suffrage Universel durant la législature 1910 - 1914. Ce ne sont pas moins de 11 rapports que le député franc-maçon de la Seine a produits avec un «art d'accommoder les restes électoraux » qui ne s'est pas démenti au fil des débats. Or celui-ci fait voir des propriétés qui retiennent l'attention du point de vue des formes de spécialisation qu'induit l'activité parlementaire. Sa collaboration avec Jaurès incarne tout d'abord la division du travail partisan socialiste entre les deux hommes : Groussier s'attachant au travail de commission en tant que rapporteur et Jaurès se concentrant sur les interventions en séance plénière à des fins de positionnement du parti. Jaurès développe d'ailleurs une théorie ad hoc du parti fondée sur une forte croyance dans sa capacité représentative : «Avec la RP, l'ordre de présentation des candidats sera l'ordre de préférence des électeurs à condition que ceux-ci soient membres vigilants de leur parti. Aujourd'hui la liberté de l'électeur est entre les mains des comités de fortune qui sous le nom de congrès tiennent la place de comités locaux impuissants. ... Ou s'organiser ou périr $»^{40}$. Membre de la CSU, Jaurès n'hésite même pas, quand sa proposition majeure sur l'utilisation inter-départementale des restes n'est pas acceptée par la commission, à la proposer à nouveau en séance plénière sous forme d'amendement ${ }^{41}$. La position de Groussier ne lui octroie évidemment ni la même marge d'action, ni les mêmes profits symboliques. Au contraire, pendant longtemps, la somme de ses rapports sur la RP demeure un stigmate attaché à son nom. A la suite de sa défaite de 1924, un électeur lui écrit : «J'avais de sérieuses raisons de croire ton succès certain [...] Hélas il faut se rendre à la réalité : tu succombes victime des divisions, des listes multiples et d'un mode de scrutin dont tu es un peu le père, mais qui a été si largement défiguré qu'il est devenu odieux à peu prés à tout le

\footnotetext{
${ }^{39}$ AN, C7447, 19 Octobre 1910.

${ }^{40}$ AN, C 7447, 23 Novembre 1910.

${ }^{41}$ AN, C 7447, 5 Février 1912.
} 
monde $»^{42}$. Groussier lui-même indique en 1927 pour expliquer son refus de se (re)présenter aux élections de 1928 qui marquent un retour au scrutin d'arrondissement : «Depuis mercredi, j'ai revécu tout mon passé de scrutin d'arrondissement, j'en ai un tel dégoût que je recule, je me refuse à me replonger dans cette boue. [...] permettez moi de rester dans une génération qui avait un autre idéal ${ }^{43}$. A l'époque où le débat sur la RP revêt une visibilité sans précédent, A. Groussier est également rapporteur de la commission du travail au sujet du projet gouvernemental de convention collective ${ }^{44}$. Bénéficiant également d'une forte mobilisation collective extra-parlementaire, la loi du 25 Mars 1919 qui institue la convention collective est marquée rétrospectivement par ces couronnements ultérieurs. La loi du 12 Juillet 1919 qui instaure elle une variante de $\mathrm{RP}^{45}$ est de son côté atteinte dans son intention supposée de stabiliser un mode plus juste de désignation électorale pour être suspecte d'avoir favoriser des résultats contradictoires en 1919 (victoire du Bloc national) et 1924 (victoire du cartel des gauches). Par là, l'histoire au présent, en échappant aux espoirs passés de ceux qui se sont imaginés faire l'avenir peut sanctionner de façon imprévisible même ceux auxquels elle a pu initialement donner une chance. Parallèlement, la conjoncture 14 - 18 a également transformé les relations de pouvoir et donc les conditions d'accès aux positions de pouvoir des membres les plus conciliateurs de la CSU aux portes de la consécration gouvernementale ${ }^{46}$.

En visant à codifier les règles de la compétition inter - partisane, la Commission du Suffrage Universel s'est opposée frontalement à l'inextricable mouvance des «représentations de la représentation » propre à une conjoncture de structuration des milieux partisans où la division du travail militant n'apparaissait pas (encore) clairement aux principaux protagonistes. En d'autres termes, si les membres de la commission qui ont siégé entre 1902 et 1919 (soit 115 députés) ${ }^{47}$ donnent une image socialement et culturellement adéquate du renouvellement du personnel parlementaire de la $3^{\text {ème }}$ république, on remarquera

\footnotetext{
${ }^{42}$ Centre d'Histoire Sociale du $20{ }^{\text {ème }}$ siècle, Université Paris I, Archives Arthur Groussier, Carton 42, «élections législatives 1919-1928».

${ }^{43}$ Ibid, lettre sans date précise mais commentant l'élection de 1924.

${ }^{44}$ Claude Didry, Naissance de la convention collective. Débats juridiques et luttes sociales en France au début du 20 è siècle, Paris, CNRS, 2000 ; sur le rôle d'A. Groussier, voir p 187 - 197.

${ }^{45} \mathrm{Il}$ s'agit en fait d'un scrutin de liste départemental à 1 tour où la proportionnelle ne joue que pour les candidats non élus à la majorité absolue.

${ }^{46}$ Il faudrait à cet effet revenir sur certains principes mis en avant par C. Charle dans sa «micro-histoire sociale des dirigeants » à propos du déclenchement du conflit. Voir Christophe Charle, La crise des sociétés impériales 1900 - 1940. France, Angleterre, Allemagne, Paris, Seuil, 2001, p 235 et suivantes.

${ }^{47}$ Ces indications sont issues de la constitution d'une base de données sur les 115 membres de la Commission du Suffrage Universel (à partir de plusieurs dictionnaires biographiques dont ceux publiés autour de l'enquête du Centre de Recherches en Histoire du $19^{\text {ème }}$ siècle - Paris I et IV).
} 
qu'ils se différencient nettement en ce qui concerne les conditions d'acquisition et de gestion de leur capital politique. Ils sont élus plus jeunes que d'autres parlementaires : $50 \%$ des membres ont été élus avant 40 ans contre, au mieux, 38\% des parlementaires de la Seine ${ }^{48}$. Mais surtout, c'est au niveau de la durée des carrières parlementaires que la différence est frappante : 60\% des commissaires ont accompli 4 mandats ou plus contre seulement 20\% pour un échantillon de députés de toute la $3^{\text {ème }}$ République $^{49}$. Ces indications appellent bien des précisions prosopographiques pour comprendre, dans la ligne des travaux de C. Charle, comment le sens de ce qui distingue ces députés d'autrui les engage à se faire une idée particulière de la délégation. Quoiqu'il en soit, ces observations élémentaires suffisent à faire toucher du doigt à quel point le travail de la CSU requiert, sous l'effet d'un sens pratique renouvelé de l'élection, de rendre public une philosophie nouvelle de la représentation. Dans ce qui apparaît comme la version la plus aboutie de l'action de la commission, c'est-à-dire l'émergence parallèle d'un Groupe Parlementaire de la Réforme Electorale, on trouve d'ailleurs une trace active et visible de ces conditions spécifiques de la délégation. En effet, le répertoire d'action choisi par le Groupe entre 1907 et 1912 (des séries de conférences de vulgarisation des fondements et des applications possibles de la RP) ne fait que redoubler la délégation qui se trouve être au fondement de la situation politique des députés conférenciers.

La mise en application des «protocoles» de cette analyse électorale qui a progressivement émergé comme le point de ralliement de l'ensemble des protagonistes parlementaires du débat entérine en ce sens les modifications sociales de la représentation politique en même temps qu'elle ne se réduit pas aux effets attendus par ses protagonistes. En s'inscrivant dans la tradition de perfectionnement des outils de compréhension de la vie politique, qui sont en même temps des outils d'action, l'analyse électorale qui passe comme en contrebande de la controverse menée par le biais de ces réunions publiques va se loger immédiatement dans les intérêts politiques de la délégation ... tout en s'écartant des intérêts politiques les plus immédiats et partisans. C'est ce qu'indique un éditorialiste du Nord en annonçant l'une de ces réunions : «C'est donc une lourde tâche que celle qui est imposée aux défenseurs de la RP et qui consiste à défendre une cause juste vis-à-vis de juges injustes [...]

\footnotetext{
48 Odile Sassi et Arlette Schweitz, "Tableaux et graphiques», dans Jean Marie Mayeur (dir.), Les parlementaires de la Seine sous la $3^{\text {ème }}$ République, Vol 1 : Etudes, Paris, Publications de la Sorbonne, 2001, p 275.

${ }^{49}$ C. Charle, «Les parlementaires: avant garde ou arrière garde d'une société en mouvement? Vue d'ensemble », dans Jean Marie Mayeur, Jean Pierre Chaline et Alain Corbin (dir.), Les parlementaires de la troisième République, Paris, Publications de la Sorbonne, 2003, p 55.
} 
Pour faire bonne figure, il est nécessaire qu'ils se sentent soutenus par l'opinion publique et qu'avant de pénétrer dans cette grotte du chien qu'est le parlement, où l'on ne respire qu'un air méphitique, ils puissent remplir leurs poumons du grand air du dehors $»^{50}$. Bien que se présentant comme «porteurs de savoirs $»^{51}$, et donc comme militants désintéressés, les députés apparaissent ainsi avoir conduit une action collective parce qu'ils étaient les seuls porteurs légitimes de représentations de leur propre activité de représentation. Le travail de transformation parlementaire des outils de cette analyse électorale écrite et savante, produits par d'autres agents du champ politique, forme donc la base de prises de parole déléguées au nom même de la mise en perspective des principes de la délégation électorale. L'institution parlementaire apparaît finalement, à travers le regroupement de certains de ses membres dans différentes activités (commission, groupe), comme une entreprise de redéfinition des images publiques de la délégation et non comme le simple reflet d'un débat intellectuel à son propos.

\footnotetext{
${ }^{50}$ La Dépêche. Journal quotidien du Nord, 31 Janvier 1909.

${ }^{51}$ Yves Gingras, Peter Keating et Camille Limoges, Du scribe au savant : les porteurs de savoir de l'Antiquité à la révolution industrielle, Paris, PUF, 2000.
} 